\title{
On a subclass of analytic functions involving harmonic means
}

\author{
Andreea-Elena Tudor and Dorina Răducanu
}

\begin{abstract}
In the present paper, we consider a generalised subclass of analytic functions involving arithmetic, geometric and harmonic means. For this function class we obtain an inclusion result, Fekete-Szegö inequality and coefficient bounds for bi-univalent functions.
\end{abstract}

\section{Introduction}

Let $U_{r}=\{z \in \mathbb{C}:|z|<r\} \quad(r>0)$ and let $U=U_{1}$ denote the unit disk.

Let $\mathcal{A}$ be the class of all analytic functions $f$ in $U$ of the form:

$$
f(z)=z+\sum_{n=2}^{\infty} a_{n} z^{n}, \quad z \in U .
$$

Further, by $\mathcal{S}$ we shall denote the class of all functions in $\mathcal{A}$ which are univalent in $U$. It is known (see [6]) that if $f \in \mathcal{S}$, then $f(U)$ contains the disk $\left\{|w|<\frac{1}{4}\right\}$. Here $\frac{1}{4}$ is the best possible constant known as the Koebe constant for $\mathcal{S}$. Thus every univalent function $f$ has an inverse $f^{-1}$ defined on some disk containing the disk $\left\{|w|<\frac{1}{4}\right\}$ and satisfying:

$$
\begin{array}{ll}
f^{-1}(f(z))=z, & z \in U \text { and } \\
f\left(f^{-1}(w)\right)=w, & |w|<r_{0}(f), r_{0}(f) \geq \frac{1}{4},
\end{array}
$$

Key Words: Analytic functions, Fekete-Szegö inequality, bi-univalent functions.

2010 Mathematics Subject Classification: 30C45.

Received: 30 April, 2014.

Revised: 20 May, 2014.

Accepted: 29 June, 2014. 
where

$$
f^{-1}(w)=w-a_{2} w^{2}+\left(2 a_{2}^{2}-a_{3}\right) w^{3}-\left(5 a_{2}^{3}-5 a_{2} a_{3}+a_{4}\right) w^{4}+\ldots
$$

We denote by $\mathcal{S}^{*}$ the class of analytic functions which are starlike in $U$.

Let $f \in \mathcal{A}$ and $\alpha, \beta \in \mathbb{R}$. We define the following function:

$$
F(z)=\left[f(z)^{1-\alpha}\left(z f^{\prime}(z)\right)^{\alpha}\right]^{1-\beta} \cdot\left[(1-\alpha) f(z)+\alpha z f^{\prime}(z)\right]^{\beta}, \quad \alpha, \beta \in \mathbb{R} .
$$

Remark 1. It is easy to observe that for specific values of $\beta$, the function $F(z)$ reduces to some generalised means. If $\beta=0$ we obtain generalised geometric means, if $\beta=1$ we obtain generalised arithmetic means and if $\beta=-1$ we obtain generalised harmonic means of functions $f(z)$ and $z f^{\prime}(z)$.

Definition 1. A function $f \in \mathcal{A}$ is said to be in the class $H_{\alpha, \beta}, \alpha, \beta \in \mathbb{R}$, if the function $F(z)$ defined by (3) is starlike, that is

$$
\Re\left\{(1-\beta)\left[(1-\alpha) \frac{z f^{\prime}(z)}{f(z)}+\alpha\left(1+\frac{z f^{\prime \prime}(z)}{f^{\prime}(z)}\right)\right]+\beta \frac{z f^{\prime}(z)+\alpha z^{2} f^{\prime \prime}(z)}{(1-\alpha) f(z)+\alpha z f^{\prime}(z)}\right\}>0, \quad z \in U .
$$

In order to prove our main results we will need the following lemmas.

Lemma 1. [9, p.24] Let $q \in Q$, with $q(0)=a$, and let $p(z)=a+a_{n} z^{n}+\cdots$ be analytic in $U$ with $p(z) \not \equiv a$ and $n \geq 1$. If $p$ is not subordinate to $q$ then there exist $z_{0}=r_{0} e^{i \theta_{0}} \in U$ and $\zeta_{0} \in \partial U \backslash E(q)$ and $m \geq n \geq 1$ for which $p\left(U_{r_{0}}\right) \subset q(U)$ and:

1. $p\left(z_{0}\right)=q\left(\zeta_{0}\right)$,

2. $z_{0} p^{\prime}\left(z_{0}\right)=m \zeta_{0} q^{\prime}\left(\zeta_{0}\right)$,

3. $\Re \frac{z_{0} p^{\prime \prime}\left(z_{0}\right)}{p^{\prime}\left(z_{0}\right)}+1 \geq m \Re\left[\frac{\zeta_{0} q^{\prime \prime}\left(\zeta_{0}\right)}{q^{\prime}\left(\zeta_{0}\right)}+1\right]$.

Denote by $\mathcal{P}$ the class of analytic functions $p$ normalized by $p(0)=1$ and having positive real part in $U$.

Lemma 2. [6] Let $p \in \mathcal{P}$ be of the form $p(z)=1+p_{1} z+p_{2} z^{2}+\ldots, z \in U$. Then the following estimates hold

$$
\left|p_{n}\right| \leq 2, n=1,2, \ldots
$$

Lemma 3. [4] If $p \in \mathcal{P}$ is of the form $p(z)=1+p_{1} z+p_{2} z^{2}+\ldots, z \in U$. Then

$$
\left|p_{2}-v p_{1}^{2}\right| \leq \begin{cases}-4 v+2, & v \leq 0 \\ 2, & 0 \leq v \leq 1 \\ 4 v-2, & v \geq 1\end{cases}
$$


When $v<0$ or $v>1$, the equality holds if and only if $p_{1}(z)=\frac{1+z}{1-z}$ or one of its rotations. If $0<v<1$ then the equality holds if and only if $p_{1}(z)=\frac{1+z^{2}}{1-z^{2}}$ or one of its rotations. If $v=0$, the equality holds if and only if

$$
p_{1}(z)=\left(\frac{1}{2}+\frac{1}{2} \lambda\right) \frac{1+z}{1-z}+\left(\frac{1}{2}-\frac{1}{2} \lambda\right) \frac{1-z}{1+z}, \quad \lambda \in[0,1]
$$

or one of its rotations. If $v=1$, the equality holds if and only if $p_{1}$ is the reciprocal of one of the functions such that the equality holds in the case of $v=0$.

\section{Inclusion result}

In this section we show that the new class $H_{\alpha, \beta}$ is a subclass of the class of starlike functions.

Theorem 1. Let $\alpha, \beta \in \mathbb{R}$ such that $\alpha \beta(1-\alpha) \geq 0$. Then

$$
H_{\alpha, \beta} \subset \mathcal{S}^{*} \subset \mathcal{S} \text {. }
$$

Proof. Let $f$ be in the class $H_{\alpha, \beta}$ and let $p(z)=\frac{z f^{\prime}(z)}{f(z)}$. Then from (4) we obtain that $f \in H_{\alpha, \beta}$ if and only if

$$
\Re\left\{\alpha(1-\beta) \frac{z p^{\prime}(z)}{p(z)}+\alpha \beta \frac{z p^{\prime}(z)}{1-\alpha+\alpha p(z)}\right\}>0 .
$$

Let

$$
q(z)=\frac{1+z}{1-z}=1+q_{1} z+\cdots .
$$

Then $\Delta=q(\mathbb{D})=\{w: \Re w>0\}, q(0)=1, E(q)=\{1\}$ and $q \in Q$. To prove that $f \in \mathcal{S}^{*}$ it is enough to show that

$$
\Re\left\{\alpha(1-\beta) \frac{z p^{\prime}(z)}{p(z)}+\alpha \beta \frac{z p^{\prime}(z)}{1-\alpha+\alpha p(z)}\right\}>0 \Rightarrow p(z) \prec q(z) .
$$

Suppose that $p(z) \nprec q(z)$.Then, from Lemma 1,there exist a point $z_{0} \in U$ and a point $\zeta_{0} \in \partial U \backslash\{1\}$ such that $p\left(z_{0}\right)=q\left(\zeta_{0}\right)$ and $\Re p(z)>0$ for all $z \in U_{\left|z_{0}\right|}$. This implies that $\Re p\left(z_{0}\right)=0$, therefore we can choose $p\left(z_{0}\right)$ of the form $p\left(z_{0}\right):=i x$, where $x$ is a real number. Due to symmetry, it is sufficient to consider only the case where $x>0$. We have

$$
\zeta_{0}=q^{-1}\left(p\left(z_{0}\right)\right)=\frac{p\left(z_{0}\right)-1}{p\left(z_{0}\right)+1},
$$


then $z_{0} p^{\prime}\left(z_{0}\right)=m \zeta_{0} q^{\prime}\left(\zeta_{0}\right)=-m\left(x^{2}+1\right):=y$, where $y<0$.

Thus, we obtain:

$$
\begin{gathered}
\Re\left[\alpha(1-\beta) \frac{z_{0} p^{\prime}\left(z_{0}\right)}{p\left(z_{0}\right)}\right]+\Re\left[\alpha \beta \frac{z_{0} p^{\prime}\left(z_{0}\right)}{1-\alpha+\alpha p\left(z_{0}\right)}\right]= \\
=\Re\left[\alpha(1-\beta) \frac{y}{i x}\right]+\Re\left[\frac{\alpha \beta y}{1-\alpha+\alpha i x}\right]=0+\frac{y[\alpha \beta(1-\alpha)]}{|1-\alpha+\alpha i x|^{2}} \leq 0 .
\end{gathered}
$$

This contradicts the hypothesis of the theorem, therefore $p \prec q$ and the proof of Theorem 1 is complete.

\section{Fekete-Szegö problem}

In 1933 M. Fekete and G. Szegö obtained sharp upper bounds for $\left|a_{3}-\mu a_{2}^{2}\right|$ for $f \in S$ and $\mu$ real number. For this reason, the determination of sharp upper bounds for the non-linear functional $\left|a_{3}-\mu a_{2}^{2}\right|$ for any compact family $\mathbb{F}$ of functions $f \in \mathcal{A}$ is popularly known as the Fekete-Szegö problem for $\mathbb{F}$. For different subclasses of $S$, the Fekete-Szegö problem has been investigated by many authors ( see [2], [4], [11]).

In this section we will solve the Fekete-Szegö problem for the class $H_{\alpha, \beta}$, where $\alpha$ and $\beta$ are positive real numbers.

Theorem 2. Let $\alpha, \beta, \mu$ be positive real numbers. If the function $f$ given by (1) belongs to the class $H_{\alpha, \beta}$, then

$$
\left|a_{3}-\mu a_{2}^{2}\right| \leq \begin{cases}\frac{-4 \mu}{(1+\alpha)^{2}}+\frac{2(\alpha-1)(1+\alpha \beta)+(\alpha+1)(5+\alpha)}{(1+2 \alpha)(1+\alpha)^{2}} & , \quad \mu \leq \sigma_{1}, \\ \frac{1}{1+2 \alpha} & , \quad \sigma_{1} \leq \mu \leq \sigma_{2}, \\ \frac{4 \mu}{(1+\alpha)^{2}}-\frac{2(\alpha-1)(1+\alpha \beta)+(\alpha+1)(3-\alpha)}{(1+2 \alpha)(1+\alpha)^{2}} & , \quad \mu \geq \sigma_{2} .\end{cases}
$$

where

$$
\sigma_{1}=\frac{1+3 \alpha-\alpha \beta+\alpha^{2} \beta}{2(1+2 \alpha)}, \quad \sigma_{2}=\frac{2+5 \alpha+\alpha^{2}-\alpha \beta+\alpha^{2} \beta}{2(1+2 \alpha)}
$$

Proof. Let $f$ be in the class $H_{\alpha}, \beta$ and let $p \in \mathcal{P}$. From (4) we obtain

$$
\left\{(1-\beta)\left[(1-\alpha) \frac{z f^{\prime}(z)}{f(z)}+\alpha\left(1+\frac{z f^{\prime \prime}(z)}{f^{\prime}(z)}\right)\right]+\beta \frac{z f^{\prime}(z)+\alpha z^{2} f^{\prime \prime}(z)}{(1-\alpha) f(z)+\alpha z f^{\prime}(z)}\right\}=p(z) .
$$

Since $f$ has the Taylor series expansion (1) and $p(z)=1+p_{1} z+p_{2} z^{2}+\ldots, z \in$ $U$, we have

$$
\begin{array}{r}
1+(1+\alpha) a_{2} z+\left[2(1+2 \alpha) a_{3}-\left(1+3 \alpha-\alpha \beta+\alpha^{2} \beta\right) a_{2}^{2}\right] z^{2}+\ldots= \\
=1+p_{1} z+p_{2} z^{2}+\ldots
\end{array}
$$


Therefore, equating the coefficients of $z^{2}$ and $z^{3}$ in (7), we obtain

$$
a_{2}=\frac{p_{1}}{1+\alpha}, \quad a_{3}=\frac{1}{2(1+2 \alpha}\left[p_{2}+\frac{\left(1+3 \alpha-\alpha \beta+\alpha^{2} \beta\right) p_{1}^{2}}{(1+\alpha)^{2}}\right] .
$$

So, we have

$$
a_{3}-\mu a_{2}^{2}=\frac{1}{2(1+2 \alpha)}\left(p_{2}-v p_{1}^{2}\right)
$$

where

$$
v=\frac{2(1+2 \alpha)}{(1+\alpha)^{2}} \mu-\frac{1+3 \alpha-\alpha \beta+\alpha^{2} \beta}{(1+\alpha)^{2}} .
$$

Now, our result follows as an application of Lemma 3.

\section{Subclass of bi-univalent function}

A function $f \in \mathcal{A}$ is said to be bi-univalent in $U$ if both $f$ and $f^{-1}$ are univalent in $U$. Let $\sigma$ be the class of all functions $f \in \mathcal{S}$ such that the inverse function $f^{-1}$ has an univalent analytic continuation to $\{|w|<1\}$. The class $\sigma$, called the class of bi-univalent functions, was introduced by Levin [7] who showed that $\left|a_{2}\right|<1.51$. Branan and Clunie [3] conjectured that $\left|a_{2}\right| \leq \sqrt{2}$. On the other hand, Netanyahu [10] showed that $\max _{f \in \sigma}\left|a_{2}\right|=\frac{4}{3}$. Several authors have studied similar problems in this direction (see [1] [5], [8], [12], [13]).

We notice that the class $\sigma$ is not empty. For example, the following functions are members of $\sigma$ :

$$
z, \frac{z}{1-z},-\log (1-z), \frac{1}{2} \log \frac{1+z}{1-z} .
$$

However, the Koebe function is not a member of $\sigma$. Other examples of univalent functions that are not in the class $\sigma$ are

$$
z-\frac{z^{2}}{2}, \frac{z}{1-z^{2}}
$$

In the sequel we assume that $\varphi$ is an analytic function with positive real part in the unit disk $U$, satisfying $\varphi(0)=1, \varphi^{\prime}(0)>0$ and such that $\varphi(U)$ is symmetric with respect to the real axis. Assume also that:

$$
\varphi(z)=1+B_{1} z+B_{2} z^{2}+\ldots, \quad B_{1}>0 .
$$


Definition 2. A function $f \in \mathcal{A}$ is said to be in the class $H_{\alpha, \beta}(\varphi), \alpha \in$ $[0,1], \beta \geq 0$, if $f \in \sigma$ and satisfies the following conditions:

$$
(1-\beta)\left[(1-\alpha) \frac{z f^{\prime}(z)}{f(z)}+\alpha\left(1+\frac{z f^{\prime \prime}(z)}{f^{\prime}(z)}\right)\right]+\beta \frac{z f^{\prime}(z)+\alpha z^{2} f^{\prime \prime}(z)}{(1-\alpha) f(z)+\alpha z f^{\prime}(z)} \prec \varphi(z),
$$

and

$(1-\beta)\left[(1-\alpha) \frac{w g^{\prime}(w)}{g(w)}+\alpha\left(1+\frac{w g^{\prime \prime}(w)}{g^{\prime}(w)}\right)\right]+\beta \frac{w g^{\prime}(w)+\alpha w^{2} g^{\prime \prime}(w)}{(1-\alpha) g(w)+\alpha w g^{\prime}(w)} \prec \varphi(w)$,

where $g$ is the extension of $f^{-1}$ to $U$.

Theorem 3. If $f \in H_{\alpha, \beta}(\varphi)$ is in $\mathcal{A}$ then

$$
\left|a_{2}\right| \leq \frac{|\tau| B_{1} \sqrt{B_{1}}}{\sqrt{\left|[(1+\alpha)+\alpha \beta(1-\alpha)] B_{1}^{2}-\left(B_{2}-B_{1}\right)(1+\alpha)^{2}\right|}},
$$

and

$$
\left|a_{3}\right| \leq B_{1}\left[\frac{1}{1+\alpha}+\frac{\alpha \beta(1-\alpha)}{2(1+2 \alpha)(1+\alpha)}\right]+\frac{\left|B_{2}-B_{1}\right|}{1+\alpha} .
$$

Proof. Let $f \in H_{\alpha, \beta}(\varphi)$ and $g=f^{-1}$. Then there exist two analytic functions $u, v: U \rightarrow U$ with $u(0)=v(0)=0$ such that:

$$
\begin{aligned}
& (1-\beta)\left[(1-\alpha) \frac{z f^{\prime}(z)}{f(z)}+\alpha\left(1+\frac{z f^{\prime \prime}(z)}{f^{\prime}(z)}\right)\right]+\beta \frac{z f^{\prime}(z)+\alpha z^{2} f^{\prime \prime}(z)}{(1-\alpha) f(z)+\alpha z f^{\prime}(z)}=\varphi(u(z)) \text { and } \\
& (1-\beta)\left[(1-\alpha) \frac{w g^{\prime}(w)}{g(w)}+\alpha\left(1+\frac{w g^{\prime \prime}(w)}{g^{\prime}(w)}\right)\right]+\beta \frac{w^{\prime}(w)+\alpha w^{2} g^{\prime \prime}(w)}{(1-\alpha) g(w)+\alpha w g^{\prime}(w)}=\varphi(v(w)) .
\end{aligned}
$$

Define the functions $p$ and $q$ by

$$
p(z)=\frac{1+u(z)}{1-u(z)}=1+p_{1} z+p_{2} z^{2}+\ldots, q(z)=\frac{1+v(z)}{1-v(z)}=1+q_{1} z+q_{2} z^{2}+\ldots
$$

or equivalently,

$$
u(z)=\frac{p(z)-1}{p(z)+1}=\frac{1}{2}\left[p_{1} z+\left(p_{2}-\frac{p_{1}^{2}}{2} z^{2}\right)+\ldots\right],
$$

and

$$
v(z)=\frac{q(z)-1}{q(z)+1}=\frac{1}{2}\left[q_{1} z+\left(q_{2}-\frac{q_{1}^{2}}{2} z^{2}\right)+\ldots\right]
$$


We observe that $p, q \in \mathcal{P}$ and, in view of Lemma 2, we have that $\left|p_{n}\right| \leq 2$ and $\left|q_{n}\right| \leq 2$, for $n \geq 1$.

Further, using (13) and (14) together with (9), it is evident that

$$
\varphi(u(z))=1+\frac{1}{2} B_{1} p_{1} z+\left(\left[\frac{1}{2} B_{1}\left(p_{2}-\frac{1}{2} p_{1}^{2}\right)+\frac{1}{4} B_{2} p_{1}^{2}\right] z^{2}+\ldots\right.
$$

and

$$
\varphi(v(z))=1+\frac{1}{2} B_{1} q_{1} z+\left[\frac{1}{2} B_{1}\left(q_{2}-\frac{1}{2} q_{1}^{2}\right)+\frac{1}{4} B_{2} q_{1}^{2}\right] z^{2}+\ldots
$$

Therefore, in view of (12), (15) and (16) we have

$$
\begin{aligned}
& (1-\beta)\left[(1-\alpha) \frac{z f^{\prime}(z)}{f(z)}+\alpha\left(1+\frac{z f^{\prime \prime}(z)}{f^{\prime}(z)}\right)\right]+\beta \frac{z f^{\prime}(z)+\alpha z^{2} f^{\prime \prime}(z)}{(1-\alpha) f(z)+\alpha z f^{\prime}(z)} \\
& =1+\frac{1}{2} B_{1} p_{1} z+\left(\frac{1}{2} B_{1}\left(p_{2}-\frac{1}{2} p_{1}^{2}\right)+\frac{1}{4} B_{2} p_{1}^{2}\right) z^{2}+\ldots
\end{aligned}
$$

and

$$
\begin{gathered}
(1-\beta)\left[(1-\alpha) \frac{w g^{\prime}(w)}{g(w)}+\alpha\left(1+\frac{w g^{\prime \prime}(w)}{g^{\prime}(w)}\right)\right]+\beta \frac{w g^{\prime}(w)+\alpha w^{2} g^{\prime \prime}(w)}{(1-\alpha) g(w)+\alpha w g^{\prime}(w)} \\
=1+\frac{1}{2} B_{1} q_{1} w+\left(\frac{1}{2} B_{1}\left(q_{2}-\frac{1}{2} q_{1}^{2}\right)+\frac{1}{4} B_{2} q_{1}^{2}\right) w^{2}+\ldots
\end{gathered}
$$

Since $f \in \sigma$ has the Taylor series expansion (1) and $g=f^{-1}$ the series expansion (2), we have

$$
\begin{aligned}
& (1-\beta)\left[(1-\alpha) \frac{z f^{\prime}(z)}{f(z)}+\alpha\left(1+\frac{z f^{\prime \prime}(z)}{f^{\prime}(z)}\right)\right]+\beta \frac{z f^{\prime}(z)+\alpha z^{2} f^{\prime \prime}(z)}{(1-\alpha) f(z)+\alpha z f^{\prime}(z)} \\
= & 1+(1+\alpha) a_{2} z+\left[2(1+2 \alpha) a_{3}-\left(1+3 \alpha-\alpha \beta+\alpha^{2} \beta\right) a_{2}^{2}\right] z^{2}+\ldots,
\end{aligned}
$$

and

$$
\begin{aligned}
& (1-\beta)\left[(1-\alpha) \frac{w g^{\prime}(w)}{g(w)}+\alpha\left(1+\frac{w g^{\prime \prime}(w)}{g^{\prime}(w)}\right)\right]+\beta \frac{w g^{\prime}(w)+\alpha w^{2} g^{\prime \prime}(w)}{(1-\alpha) g(w)+\alpha w g^{\prime}(w)} \\
= & 1-(1+\alpha) a_{2} w-\left[2(1+2 \alpha) a_{3}-\left(1+3 \alpha-\alpha \beta+\alpha^{2} \beta\right)\left(a_{3}-2 a_{2}^{2}\right)\right] w^{2}+\ldots
\end{aligned}
$$


Equating the coefficients in (17), (19) and (18), (20), we obtain

$$
\left\{\begin{array}{l}
(1+\alpha) a_{2}=\frac{1}{2} B_{1} p_{1}, \\
2(1+2 \alpha) a_{3}-\left(1+3 \alpha-\alpha \beta+\alpha^{2} \beta\right) a_{2}^{2}=\frac{1}{2} B_{1}\left(p_{2}-\frac{p_{1}^{2}}{2}\right)+\frac{1}{4} B_{2} p_{1}^{2}, \\
-(1+\alpha) a_{2}=\frac{1}{2} B_{1} q_{1} \\
-2(1+2 \alpha)\left(a_{3}-2 a_{2}^{2}\right)-\left(1+3 \alpha-\alpha \beta+\alpha^{2} \beta\right) a_{2}^{2}=\frac{1}{2} B_{1}\left(q_{2}-\frac{q_{1}^{2}}{2}\right)+\frac{1}{4} B_{2} q_{1}^{2} .
\end{array}\right.
$$

From the first and the third equation of the system (21) it follows that

$$
p_{1}=-q_{1},
$$

and

$$
a_{2}^{2}=\left(\frac{B_{1} p_{1} \tau}{4(1+\gamma)}\right)^{2}
$$

Now, (22), (23) and the next two equations of the system (21) lead to

$$
a_{2}^{2}=\frac{B_{1}^{3}\left(p_{2}+q_{2}\right)}{4[(1+\alpha)+\alpha \beta(1-\alpha)] B_{1}^{2}-4\left(B_{2}-B_{1}\right)(1+\alpha)^{2}} .
$$

Thus, in view of Lemma 2, we obtain the desired estimation of $\left|a_{2}\right|$.

From the third and the fourth equation of (21), we obtain

$a_{3}=\frac{1}{2} B_{1} p_{2} \frac{3+5 \alpha+\alpha \beta(1-\alpha)}{4(1+2 \alpha)(1+\alpha)}+\frac{1+3 \alpha-\alpha \beta(1-\alpha)}{4(1+2 \alpha)(1+\alpha)}+\frac{1}{4} p_{1}^{2}\left(B_{2}-B_{1}\right) \frac{1}{1+\alpha}$,

which yields to the estimate given by (11) and so the proof of Theorem 3 is completed.

\section{References}

[1] R. M. Ali, S. K. Lee, V. Ravichandran, S. Supramanian, Coefficient estimates for bi-univalent Ma-Minda starlike and convex functions, Appl. Math. Lett. 25(2012), 344-351.

[2] D. Bansal, Fekete-Szegö problem for a new class of analytic functions, Int. J. Math. Mathematical Sciences 2011(2011), article ID: 143096, 5 pages. 
[3] D. A. Branan, J. G. Clunie, W. E. Kirwan, Coefficient estimates for a class of starlike functions, Canad. J. Math. 22(1970), 476-485.

[4] M. Fekete, G. Szegö, Eine Bemerkung über ungerade schlichte Functionen, J. London Math. Soc. 8(1933) , 85-89

[5] S. P. Goyal, P. Goswami, Estimate for initial Maclaurin coefficients of biunivalent functions for a class defined by fractional derivatives, J. Egyptian Math. Soc. 20(2012), issue 3,179-182.

[6] A. W. Goodman, 'Univalent functions', Polygonal Publishing House, Washington, New Jersey, 1983.

[7] M. Lewin, On a coefficient problem for bi-univalent functions, Proc. Amer. Math. Soc. 18(1967), 63-68.

[8] X.-F. Li, A.-P. Wang, Two new subclasses of bi-univalent functions, Int. Math. Forum no. 30, 7(2012), 1495-1504.

[9] S.S. Miller, P.T. Mocanu, 'Differential Subordinations: Theory and Applications', Dekker, New York, 2000.

[10] E. Netanyahu, The minimal distance of the image boundary from the origin and the second coefficient of a univalent function in $|z|<1$, Arch. Ration. Mech. Anal. 32(1969), 100-112.

[11] H. M. Srivastava, A. K. Mishra, M. K. Das, The Fekete-Szegö problem for a subclass of close-to-convex functions, Complex Variables, Theory Appl. 44(2001), 145-163.

[12] H. M. Srivastava, A. K. Mishra, P. Gochhayat, Certain subclasses of analytic and bi-univalent functions, Appl. Math. Lett. 23(2010), 1188-1192.

[13] Q.-H. Xu, Y.-C. Gui, H.M. Srivastava, Coefficient estimates for a certain subclass of analytic and bi-univalent functions, Appl. Math. Lett. 25(2012), 990-994.

Andreea-Elena TUDOR,

Department of Mathematics,

Transilvania University of Braşov,

Str.Iuliu Maniu 50, 500091, Brasov, Romania.

Email: tudor_andreea_elena@yahoo.com

Dorina RĂDUCANU,

Department of Mathematics,

Transilvania University of Braşov,

Str.Iuliu Maniu 50, 500091, Brasov, Romania.

Email: draducanu@unitbv.ro 
ON A SUBCLASS OF ANALYTIC FUNCTIONS INVOLVING HARMONIC 